DANKA, István

\title{
Which Way to Argue (For): The Visual, the Linguistic AND the SYMBolic ${ }^{1}$
}

This paper relates three notions to each other: visual/pictorial, linguistic/verbal and symbolic/formal reasoning. I shall argue that in order to find a role of visuality in reasoning in general, differences from symbolic reasoning are to be exploited rather than dissolved (as visual logicians normally intend to do). Understanding visual reasoning as complementary to, rather than interchangeable with, symbolic reasoning could better serve educational purposes. Being spatial rather than sequential, visual logic differs from symbolic logic but is also similar to at least some forms of linguistic reasoning. Visual reasoning better supports finding connections among concepts than finding proofs for claims. Hence, that would be at least one good direction towards which it should be developed.

\section{The Problem}

It is often claimed that the logical structure of (linguistic and/or formal) reasoning and that of images are incompatible (for an overview of pro and contra arguments, see Shin-LemonMumma 2014). Visual or pictorial arguments are rarely applied in reasoning, and where they are applied at all, they are treated as illustrations rather than substantial parts of argumentation. Though Aristotle still thought that visual demonstration is a method of persuasion (Blair 2008, p. 41), e.g. he discussed a visual demonstration of the Pythagorean Theorem in Metaphysics 1051a26-26 (see a classical version:

http://www.crewtonramoneshouseofmath.com/images/pythagorean-theorem.gif), except a few proponents of diagrammatic reasoning like Euler, Venn and Peirce, visual arguments were not seriously researched. A spread of symbolic or formal logic in the previous century also did not encourage logicians to develop visual representations as arguments, even though Frege's own notation was at least partly visual (imagine these samples from his Begriffschrift without the graphical part:

<http://www.stephenwolfram.com/publications/mathematical-notation-past-

future/Images/Frege.jpg>).

Visual arguments nonetheless provide a very helpful tool for representing logical relations in a clear and comprehensible way. Sun-Joo Shin, a leading figure of a recent Renaissance of diagrammatology (the study of applying diagrams for representing logical relations) extensively argues that visual representations are more natural bearers of logical relations than symbolic, and even linguistic, ones. Shin (1994) thinks that linguistic systems are conventional, whereas pictorial representation is based (primarily) on resemblance. In this sense, for her, "diagrammatic representation is more natural than linguistic representation" (Englebretsen 1996, p. 327).

1The results presented in this paper have been developed within the framework of "Integrative Argumentation Studies" supported by the Hungarian Scientific Research Fund (OTKA K-109456). 
At least if Goodman (1968)'s view that pictorial representations do not rely on resemblance but pure convention is set aside (and there are good reasons to do so - see Nyíri (2001)), it seems reasonable to claim that visual representations are more natural meaning bearers than linguistic or symbolic representations. It may be questionable though whether this applies to logical relations in particular. Due to their abstract nature, they seem to be really far from the realm of visuality. Nonetheless, basic logical relations are often introduced in logic textbooks and courses via a set theoretical approach containing pictorial representations of sets called Venn-diagrams. Insofar as at least elementary logical relations can be properly represented visually, diagrams can play a central role in introducing students into logic in a (presumably) easier way than via symbolic representations. However, after introduction, they used to be fully replaced with symbolic notation typically because "[g]enuine formal reasoning, so the claim goes, takes place in the head. Pictures may help the novice to get the right idea, but they are really incidental to the process" (Englebretsen 1996, p. 323). This is what visual logicians used to call a "general prejudice [of logicians] against diagrams" (Shin 1994, p. 1).

Hence, other than a theoretical importance of possible applications of visual representations in reasoning (see esp. Lemon-De Rijke-Shimojima eds. 1999), further reasons also call for clarifying relations between a logic of language(s) and that of images. Two of them are to be emphasised. First, the question whether "genuine reasoning" is (purely) formal at all has a philosophical importance. Second, whether reasoning can be effectively taught through visual representations at an appropriately high level so that visuality can also serve as implementing, rather than just introducing into, argumentation has an educational importance. While the first issue can of course not be properly answered here, some aspects of it will be touched upon. For the second, I hope to give a proper answer, claiming that in order to exploit its benefits, visual logic should be seen complementary to, rather than interchangeable with, symbolic logic.

\section{Pictorial and Symbolic Representations}

Diagrams differ from symbolic representations in some important sense. They are spatial (more precisely, planar or two-dimensional), whereas symbolic representations are sequential (and hence one-dimensional). Sequential representations are semantically much less rich than spatial representations and in compensation, the former requires a richer syntax. Hence, visual and symbolic representations of arguments can differ significantly in their semantic richness as well as syntactic structure. Diagrams apply an extensional (or set theoretical) approach, taking logical relations to be relations between classes and their elements. In contrast, symbolic representations normally take an intensional approach in which logical relations are considered to be relations between subjects and predicates. This difference has proved to be essential. A problem with the set theoretical approach is that "not all relations can be viewed as membership or inclusion" - an example might be polyadic predicates (Englebratsen 1996, p. 328).

While propositional logic can be well represented by diagrams, a main concern of diagrammatists from Euler via Venn and Peirce to Shin has been representing first-order predicate logic on diagrams properly. Though Shin (1994) managed to develop a system 
based on a Peircean extension of Venn-diagrams that is complete and sound on first-order predicate logic (and hence can handle existentially quantified statements, the most problematic part of Venn's system), the more effort put into developing Venn-diagrams seems to involve the least promising results.

The problem with Shin-diagrams is as follows. At least from the educational point of view I intend to defend, diagrammatic reasoning is valuable due to its comprehensibility. Diagrams demonstrate logical relations in an easy-to-grasp way. It seems, however, that by increasing the complexity of logical relations, the advantages of visual representations decrease. Namely, comprehensibility decreases more along with an increase of complexity in the case of diagrams than that of symbolic representations. A demonstration of this might be comparing a visual and a symbolic proof of the completeness and soundness of Shin's system. Hammer and Danner (1996) provided an 8-page-long visual demonstration of the completeness and soundness of Shin-diagrams. Miller (2006) presented another proof in symbolic/linguistic terms in no more than five lines (and a bit of explanation). Promoting visual clarity and aiming at logical expressibility seem to lead to opposite directions. As the complexity increases, symbolic logic is the easier-to-grasp way of representing logical relations. Hence, a role of visual representations in educating logic seems to be limited at best.

Shin-diagrams are of course scientifically valuable at their own right. But their educational usefulness is questionable if they provide a more complex and harder-to-grasp way of representing logical relations than symbolic representations do. It seems to me that developing visual logic into the direction that it should better imitate what symbolic logic does anyway (and in what symbolic logic is better) is a strategic failure. Rather than trying to dissolve their dissimilarities, they could also be exploited.

How appropriate a representation is largely depends on what we intend to represent by it. If the representandum is an abstract world, real or constructed, of ever increasingly more complex logical relations among logical entities, real or constructed, then symbolic logic is certainly the most appropriate tool among the three for representing it. If the representandum is common sense reasoning and argumentation, linguistic (or so-called informal) logic seems to be the best candidate. Finally, if the representandum is spatial relations, visual logic enjoy serious advantages. Rather than aiming at being better in representing the abstract, visual logic should aim at representing the spatial.

If symbolic logic is taken to be the par excellence form of logic, a clash between expressibility and clarity is not specific about visual representations; it applies to linguistic representations of logical relations as well (as there is no linguistic representation of e.g. higher-order logics). But no one thinks that verbal argumentative skills should be improved by developing a system of translations from complex symbolic logic to everyday verbal expressions. Why should visual logic be developed into that direction then? While in the case of symbolic logic, along with complexity, syntactic richness increases, in the case of visual and verbal argumentation, along with complexity, semantic richness increases. Building on this similarity between the visual and the verbal, visual logic should be applied for representing semantically rather than syntactically complex reasoning. Central to this, a note is to be made about the sequentiality vs. spatiality issue. 


\section{Sequentiality and Spatiality}

An argument why common-sense arguments can be better represented in symbolic than visual logic is that both symbolic logic and linguistic reasoning are sequential, whereas visual logic is spatial. As mentioned above, a consequence of this is a richer syntax of sequential representations and a richer semantics of spatial representations. But in the previous section I just have argued that by increasing complexity in linguistic representations, it is normally their semantics rather than their syntax increases (as their syntax rarely goes beyond that of a first-order modal and/or intensional logic). Even if these two claims are not in a direct contradiction, there is some conflict between them.

In order to dissolve the conflict, one may develop systems of sequential visual logic. This is what Englebretsen did before Shin (1994)'s revolutional work on Venn-diagrams when he made an attempt to develop a linear rather than planar visual representation of first-order logical relations (Englebratsen 1992, p. 37). However, Englebretsen's notion of linearity is too broad to provide a suitable response to the problem of sequentiality and spatiality. He needs a two-dimensional framework in order to relate his lines to one another. E.g. he represents the statement "Some $\mathrm{S}$ is $\mathrm{P}$ " by two lines crossing one another, standing for two sets having an intersection in Venn's system (see Englebratsen 1992, p. 39). Applying lines instead of circles does not involve sequentiality and hence does not solve the problem.

I shall rather follow another line, namely, arguing that linguistic arguments are not sequential; more precisely, the universal claim that all linguistic arguments are sequential is false. An argument presented orally is of course sequential as there is no other way of expressing it than uttering one sentence after the other. But written arguments differ from oral arguments. Core arguments in written texts are often highlighted by visually distinguishing them from the rest of the (linear) text, traditionally in the form of syllogisms. Syllogisms, in contrast with linear texts, are not sequential; they form a tree-structure. At least two reasons can support this claim. First, in fact, other than polysyllogisms, arguments are rarely (or perhaps never) sequential because in order to accept the conclusion, all premises have to be accepted and had in mind synchronously rather than one after the other as this is the reason why accepting the premises is taken to be necessary for accepting the conclusion. Second, premises (and even the conclusion in most cases) can be put into any order without hurting their logical relations to one another. Hence, their logical structure does not depend on their place in a sequence of their utterance. Arguments in linear texts may or may not be tree-structured but if the above-mentioned are right, where they are not tree-structured, they represent less properly the logical relations in question.

Trees, just as Englebretsen's diagrams, consist of lines but are nonetheless two-dimensional representations. A tree has two or more branches (premises) and a parent branch or trunk (conclusion). Complexity in tree-structured arguments does not increase via syntactical complexity but more and more (and thinner and thinner) branches and twigs supporting their parent branches by gradually finer and finer arguments. Hence, representing an argument in a tree requires at least two dimensions to develop (a depth of argumentation, i.e., gradually thinner arguments on the one hand, and a width of argumentation, i.e., more than one arguments supporting their parent branch), and also cannot meaningfully transformed to any one-dimensional representational form. If so, arguments that can be suitably represented in the form of a syllogism can better represented visually than 
symbolically (holding the supposition that symbolic logic is sequential whereas visual logic is spatial).

A possible objection may be that my claim is about the nature of arguments themselves rather than their linguistic representations. Perhaps the logical structure of arguments differ from that of their linguistic representations. I can accept that some linguistic representations of arguments are de facto linear. But not all of them are and hence sequentiality cannot be a differentia specifica of linguistic representations. Furthermore, if linguistic representations were always sequential then visual logic is even better than linguistic logic in representing tree-structured arguments (i.e., most types of syllogistic reasoning at the very least).

Texts allow the writer to construct their representations in a visually ordered manner. Spatiality, and hence visuality, occurs even within texts themselves. Forming core arguments in the form of syllogisms rather than flowing texts is a visual element in linguistic representations. Multimedia documents just broaden, though with no doubt dramatically, the possibilities of including visual elements in texts and hence written arguments - not to talk about moving pictures, being inherently spatial and sequential at the same time.

Disregarding sequences of spatial representations as a too cheap way for promoting visual logic, a direction which may also worth following is another strategic move: making an advantage of its (allegedly disadvantageous) extensional, set theoretical approach. If spatial representations are better at their semantics than syntax, an obvious way to follow would be applying visual logic to analysing semantic rather than syntactic relations like relating concepts to one another in terms of inclusion, intersection, exclusion, etc. of their extension. This can increase the potential for analysing complex arguments, insofar as complexity typically increases with the involvement of more and more complex concepts. Tree structures can also be applied for classification where inclusion-exclusion is a central aspect. Putting philosophical ideas or scientific concepts into a space of logic and relating them to one another could also help in setting up a framework for a dialectics of positions interrelated in a complex way. Visual logic may not be the best logic for proofs and deductions but it certainly could be a proper tool for identifying relations and connections among concepts and complex views which have a more-than-one-dimensional extension.

\section{Conclusion}

In this paper I have argued that in order to find a role of visuality in reasoning, differences from symbolic logic are to be exploited rather than dissolved. I have identified a strategic failure of visual logicians whose main concern is to show that visual and symbolic logic are interchangeable. Applying a perspective from educating logic, I have found that seeking for dissimilarities (in order to make the two complementary) rather than similarities (in order to translate from one to the other) is a strategically more promising way to find a place for visual logic.

I have also claimed that central to my aims, it must be accepted that a relation among visual, linguistic and symbolic reasoning depends on what is claimed to be represented by them, and an answer to that question depends on explanatory purposes rather than pure facts. This might be seen as at least a partial response to the philosophical question I have raised in 
Section One: formal reasoning is not necessarily the only, or even the most important, representandum of a logical system.

A response to the educational question has been that formal reasoning, at a certain level of complexity, should not be taught visually. But complexity could be increased in ways different from syntactical complexity, and those directions are open for visual logic. In education, logic intends to represent the way how we humans think and argue. In this respect, spatial representations of semantic relations can be extremely important. Visual reasoning is better in establishing connections among concepts than symbolic logic. Hence, if that task is attributed to it, visual logic can play a central role in logics education.

\section{References}

- Blair, J. Anthony (2008) "The Rhetoric of Visual Arguments." In (Hill, Charles A. Helmers, Marguerite eds.), Defining Visual Rhetorics. London - Mahwah (NJ): LEA Publishers.

- Englebretsen, George (1992) "Linear Diagrams for Syllogisms (with Relationals)." Notre Dame Journal of Formal Logic 33 (1): pp. 37-69.

- Englebretsen, George (1996) A review of Sun-Joo Shin, The Logical Status of Diagrams. Modern Logic 6 (3): pp. 322-330.

- Goodman, Nelson (1968) Languages of Art. Indianapolis: Bobbs-Merrill.

- Hammer, E. - Danner, N. (1996) "Towards a Model Theory of Venn Diagrams." Journal of Philosophical Logic 25 (5): pp. 463-482.

- Lemon, Oliver - De Rijke, Maarten - Shimojima, Atsushi eds. (1999) Efficacy of Diagrammatic Reasoning. A special issue of Journal of Logic, Language and Information 8 (3).

Miller, Nathaniel (2006) "A Brief Proof of the Full Completeness of Shin's Venn Diagram Proof System." Journal of Philosophical Logic 35 (3): pp. 289-291.

- Nyíri, Kristóf (2001) "The Picture Theory of Reason." In (Brogaard, Berit - Smith, Barry eds.), Rationality and Irrationality, Wien: öbv-hpt. pp.242-266. URL: http://nyitottegyetem.phil-inst.hu/filtort/kut/krb2000/tlk.htm

- Sun-Joo Shin (1994) The Logical Status of Diagrams. Cambridge: Cambridge University Press.

- Shin, Sun-Joo, Lemon, Oliver and Mumma, John (2014) "Diagrams." In (Zalta, Edward N. ed.), The Stanford Encyclopedia of Philosophy (Winter 2014 Edition). URL: <http://plato.stanford.edu/archives/win2014/entries/diagrams/> 